\title{
Sources of X-rays from galaxies
}

\author{
Q. Daniel Wang ${ }^{1}$ \\ ${ }^{1}$ Astronomy Department, University of Massachusetts, USA \\ email: wqd@astro.umass.edu
}

\begin{abstract}
Galactic X-ray emission is a manifestation of various high-energy phenomena and processes. The brightest X-ray sources are typically accretion-powered objects: active galactic nuclei and low- or high-mass X-ray binaries. Such objects with X-ray luminosities of $\gtrsim 10^{37} \mathrm{ergs} \mathrm{s}^{-1}$ can now be detected individually in nearby galaxies. The contributions from fainter discrete sources (including cataclysmic variables, active binaries, young stellar objects, and supernova remnants) are well correlated with the star formation rate or stellar mass of galaxies. The study of discrete X-ray sources is essential to our understanding of stellar evolution, dynamics, and end-products as well as accretion physics. With the subtraction of the discrete source contributions, one can further map out truly diffuse X-ray emission, which can be used to trace the feedback from active galactic nuclei, as well as from stars, both young and old, in the form of stellar winds and supernovae. The X-ray emission efficiency, however, is only about $1 \%$ of the energy input rate of the stellar feedback alone. The bulk of the feedback energy is most likely gone with outflows into large-scale galactic halos. Much is yet to be investigated to comprehend the role of such outflows in regulating the ecosystem, hence the evolution of galaxies. Even the mechanism of the diffuse X-ray emission remains quite uncertain. A substantial fraction of the emission cannot arise directly from optically-thin thermal plasma, as commonly assumed, and most likely originates in its charge exchange with neutral gas. These uncertainties underscore our poor understanding of the feedback and its interplay with the galaxy evolution.
\end{abstract}

Keywords. galaxies: general; X-rays: galaxies, binaries, ISM; stellar dynamics

\section{Introduction}

Why are X-rays from galaxies interesting? X-ray emission from a galaxy typically arises from high-energy (high temperature or relativistic) processes in extreme conditions. Such processes are important to understand in their own right, but are also believed to play a major role in shaping the structure and evolution of galaxies via various types of feedback (e.g., Tang et al. 2009; Oppenheimer et al. 2010). In particular, the X-ray emission effectively traces diffuse hot plasma, which may dominate the volume and account for the bulk of the baryon mass in and around galaxies, especially massive ones (e.g,, Crain et al. 2010). The X-ray wavelength range also contains major atomic transitions (e.g., all K-shell transitions of carbon through iron), providing key thermal, chemical, and kinematic diagnostics of the interstellar medium (ISM) in all phases (cold, warm, and hot) and all forms (atomic, molecular, and dust). X-rays are also energetic and penetrating, which may heat and evaporate dust grains/molecules and even companion stars in Xray binaries, significantly affecting stellar and interstellar radiation. X-rays can often be observed through dense clouds with column densities up to $\gtrsim 10^{24} \mathrm{~cm}^{-2}$. Therefore, Xray observations are a powerful tool in examining the stellar and interstellar properties of galaxies.

Substantial progress in understanding the X-ray emission from nearby galaxies has been made over the past decade or so. Chandra and XMM-Newton X-ray Observatories, in particular, have provided a combination of superb spatial and spectral resolutions (reaching $\sim 1^{\prime \prime}$ and $500 \mathrm{~km} \mathrm{~s}^{-1} \mathrm{FWHM}$ ) as well as large collecting areas (up to $\sim 3000$ 
$\mathrm{cm}^{2}$ ) over a broad energy range of 0.3-10 keV. These capabilities have enabled extensive studies of various classes of X-ray sources: their luminosity functions, spectral characteristics, and relationships to other galactic properties such as the star formation rate (SFR) and total stellar mass. These are the topics that I am going to review, focusing on recent results.

\section{Discrete X-ray Sources}

Active Galactic Nuclei (AGN). AGNs are powered by the accretion of matter by supermassive black holes (SMBHs) from their surroundings. This process can be very efficient, energetically, converting up to about $10 \%$ of the accreted mass into radiation and mechanical energy. For example, the energy released from the growth of a SMBH can be about 100 times the gravitational binding energy of its host galaxy. This energy feedback, if well coupled to the surrounding medium, should then have enormous impact on galaxy formation and evolution.

While much of the SMBH growth occurs at redshifts greater than 1, present AGNs typically have only moderate radiation luminosities $L_{x} \sim 10^{42}-10^{45} \mathrm{ergs} \mathrm{s}^{-1}$ and appear in only about $10 \%$ of nearby galaxies. Such AGNs are most easily identified in X-ray, since their luminosities are still considerably higher than those of other galactic sources. AGNs typically have intrinsic X-ray spectra that can be characterized by a power law with a photon index of $\sim 1.7$. However, a significant fraction of AGNs are known to be severely obscured or even Compton-thick to X-rays. Such AGNs may still be detected in reflected or reprocessed light, mostly in optical, mid-IR, and/or hard X-ray (from photonionized gas, hot dust, and/or Fe florescence; e.g., LaMassa et al. 2009). Observations of the AGNs can also provide us with a better view of their feedback effects on the immediate surroundings. Their X-ray spectra typically show a mixture of collisionally excited hot thermal plasma, characterized by numerous strong resonance lines, and photoionized plasma, represented by strong forbidden lines and recombination continuum (e.g., Guainazzi \& Bianchi 2007). Interestingly, the X-ray luminosities required to model such spectra sometimes far exceed those of the present AGNs, indicating that they may have been substantially brighter in the recent past (e.g., Wang et al. 2010) or that some other processes such as charge exchange $(\mathrm{CX})$ between hot ions and neutral atoms may need to be considered (see later discussions; Liu et al. 2011b).

When a $\mathrm{SMBH}$ is in a low- $L_{x}\left(\lesssim 10^{42}\right.$ ergs s $\left.^{-1}\right)$ or even moderate- $L_{x}$ state, the accretion is likely in the so-called radiatively inefficient (radio) mode (e.g., Yuan 2007). Much of the gravitational energy of the accreted matter may be released in form of mechanical energy (e.g., an accretion disk wind and/or jet; Omma et al. 2004). Such energy release may play an important role in regulating the nuclear environment of galaxies. A SMBH may also undergo intermittent accretion episodes, resulting in cycles of heating and cooling of surrounding gas (e.g., Yuan et al. 2009; Pellegrini et al. 2011). Various studies are ongoing to explore the interplay between the SMBH accretion and the nuclear environment of very nearby galaxies $(D \lesssim 10 \mathrm{Mpc}$; e.g., Li et al. 2009, 2011). For more distant galaxies, confusion with stellar sources typically becomes too severe to even identify individual low- $L_{x}$ AGNs with certainty, especially in galaxies with active nuclear SF.

Sources Related to Recent Star Formation. A nearby galaxy, excluding its possible AGN, typically has $L_{x} \lesssim 10^{42} \mathrm{ergs} \mathrm{s}^{-1}$, depending primarily on its mass and SFR. In an active SF galaxy, the luminosity is normally dominated by high-mass X-ray binaries (HMXBs), which contain neutron stars or stellar mass black holes accreting typically from stellar winds of companions more massive than $\sim 5 M_{\odot}$. The spectrum of such a HMXB can 
be characterized by a power law with a photon index of $\sim 1.2$, a cutoff at $\sim 20 \mathrm{keV}$, plus an Fe-K emission feature centered at $6.4-6.7 \mathrm{keV}$ and with an equivalent width of 0.2-0.6 keV (White et al. 1983). The X-ray luminosity function (XLF) of HMXBs can be characterized by a power law with a differential slope of 1.5-1.6 over a very broad luminosity range of $10^{36}-10^{40} \mathrm{ergs} \mathrm{s}^{-1}$ (Grimm et al. 2003; Persic \& Rephaeli 2007 and references therein). The XLF shows an exponential cutoff at $\sim 2 \times 10^{40} \mathrm{ergs} \mathrm{s}^{-1}$ (Swartz et al. 2011) and seems to flatten out again at $\gtrsim 10^{41} \mathrm{ergs} \mathrm{s}^{-1}$.

Much attention has been placed on so-called ultra-luminous X-ray sources (ULXs) non-nuclear, point-like, X-ray sources with apparent isotropic $L_{X} \gtrsim 10^{39} \mathrm{ergs} \mathrm{s}^{-1}$, which is roughly the Eddington luminosity of a stellar mass BH (e.g., Swartz et al. 2011 and references therein). ULXs tend to have steep spectra with a mean power law photon index of $\sim 1.7$. There is on average $\sim 1$ ULX per $0.5 \mathrm{M}_{\odot} \mathrm{yr}^{-1}$ SFR in local galaxies. ULXs are clearly a heterogeneous population of X-ray sources, including very young supernova remnants (SNRs, due to the interaction of SN ejecta with dense circumstellar materials), as well as probably mildly beamed and/or super-Eddington HMXBs. Some of these sources, especially those rare ones with $L_{X} \gtrsim 10^{41} \mathrm{ergs} \mathrm{s}^{-1}$, may represent the so-called intermediate-mass BHs (IMBHs with $10^{2} M_{\odot} \lesssim M_{B H} \lesssim 10^{4} M_{\odot}$ ) undergoing subEddington accretion. IMBHs may be formed from the collapse of Pop III stars and at centers of globular clusters (GCs) and dwarf elliptical galaxies, which may since being destroyed dynamically.

There are other young stellar objects and their remnants such as YSOs and colliding wind binaries, as well as normal SNRs. Such sources are typically quite faint individually $\left(\lesssim 10^{35} \mathrm{ergs} \mathrm{s}^{-1}\right)$; but collectively they can still account for a significant fraction of the $\mathrm{X}$-ray emission from galaxies, especially when the contribution from brighter sources has been excised: $2-10 \mathrm{keV} L_{x} \approx 10^{38.2} \operatorname{ergs~s}^{-1}\left[M_{\odot} \mathrm{yr}^{-1}\right]^{-1}$ (Bogdán \& Gilfanov 2011).

The total X-ray luminosity of young stellar objects and their remnants in a galaxy is strongly correlated with the SFR: $L_{x}=10^{39.4} \operatorname{ergs~s}^{-1}\left[\begin{array}{lll}M_{\odot} & \mathrm{yr}^{-1}\end{array}\right]^{-1}$ with a scatter of 0.4 dex (e.g., Mineo et al. 2011). With this correlation, accounting for its possible cosmological evolution (Dijkstra et al. 2011) as well as the scatter, one can estimate the SFR of a distant galaxy from its X-ray luminosity. Or if we know the SFR from its other proxies, we can then compare the predicted and observed luminosities to constrain a potential AGN contribution.

Sources from Old Stars. A galaxy with little recent SF can still contain copious X-ray sources, both discrete and diffuse. In this case, the brightest off-nuclear discrete sources are low-mass X-ray binaries (LMXBs), which contain neutron stars or stellar-mass BHs accreting from their typically post-main-sequence, Roche-lobe-overflowing companions with masses $\lesssim 1 M_{\odot}$. Therefore, LMXBs trace stellar populations older than $\sim 1$ Gyr.

The average X-ray spectrum of LMXBs can be characterized by a power law with a photon index of $\sim 1.6$, considerably steeper than that of HMXBs. LMXBs at the high end of their $0.3-10 \mathrm{keV}$ luminosity range, $\sim 10^{39} \mathrm{ergs} \mathrm{s}^{-1}$, tend to have even softer spectra and are similar to Galactic black hole X-ray binary candidates in their very high state (Irwin et al. 2003).

The XLF of LMXBs has a convex shape. It can be represented approximately by a power law with a differential slope of $\sim 2.0$ in the luminosity range $L_{X} \sim 5 \times 10^{37}-$ $5 \times 10^{38} \mathrm{ergs} \mathrm{s}^{-1}$ (Gilfanov 2004; Kim \& Fabbiano 2004). The XLF steepens at higher luminosities, which appears to be more significant with the increasing galaxy age (Kim \& Fabbiano 2010); the XLF of young elliptical galaxies (a few Gyr old) is intermediate between that of typical old elliptical galaxies and that of star-forming galaxies. The overall steepening of the XLF with the luminosity can be explained by the mass transfer in binary systems with giants, which significantly shortens their stellar lifetime (Revnivtsev et al. 
2011). In contrast, the majority of binary systems at luminosities $\lesssim 5 \times 10^{37}$ have mainsequence secondary companions (except for those with white dwarf donors), apparently responsible for the flattening of the XLF to a slope $\sim 1$ (Revnivtsev et al. 2011). The normalization of the XLF is proportional to the stellar mass, which can be estimated from the K-band luminosity using a color-based correction for the mass-to-light ratio of a galaxy (Bell \& de Jong 2001). On average, the combined luminosity of LMXBs with individual $L_{X} \gtrsim 10^{37} \mathrm{ergs} \mathrm{s}^{-1}$, which can be detected in a nearby galaxy with a reasonable exposure of Chandra or XMM-Newton, is $(8 \pm 0.5) \times 10^{39} \operatorname{ergs~s}^{-1}$ per $10^{11} M_{\odot}$ (Gilfanov 2004); the residual contribution down to $L_{x} \sim 10^{35} \mathrm{ergs} \mathrm{s}^{-1}$ (typically $\lesssim 20 \%$, especially in the $\lesssim 2 \mathrm{keV}$ band) can be well estimated with the flat XLF.

The average properties of fainter X-ray sources, mostly cataclysmic variables $(\mathrm{CVs})$ and active binaries (ABs), which are typically in the range of $10^{30}<L_{x}<10^{34}$ ergs s $^{-1}(2-10$ $\mathrm{keV}$ ), have also been measured. Sazonov et al. (2006) show that the specific luminosity of such sources (per unit stellar mass) in the solar neighborhood is $(2.0 \pm 0.8) \times 10^{27}$ and $(1.1 \pm 0.3) \times 10^{27} \mathrm{erg} \mathrm{s}^{-1} \mathrm{M}_{\odot}^{-1}$. The inferred total contribution of ABs and CVs to the 2-10 keV luminosity of the Milky Way is $\sim 2 \times 10^{38}$ ergs s$^{-1}$, or $3 \%$ of the integral luminosity of LMXBs. The specific luminosity has also been estimated for nearby lowmass early-type galaxies (M32 and NGC 3379; Revnivtsev et al. 2008) and is shown to be a factor of $\sim 2$ lower than the solar neighborhood value. The exact cause of this difference is yet to be identified. One possibility is that it represents the genuine difference in the binarity of stars, resulting from different stellar formation and evolution environments.

Indeed, the environment-dependence of the XLF is clearly present for LMXBs (e.g., Zhang et al. 2011 and references therein). The XLF shape of sources in globular clusters (GCs) is shown to be significantly different from that in the field, especially in $10^{35}-$ $10^{37}$ ergs s$^{-1}$. Over this luminosity range (relative to more luminous ones), the fraction of sources in GCs is about 4 times less than in the field. A similar result is also obtained in a recent study of the XLFs of sources over a substantially lower luminosity range (e.g., Fig. 1, Xu, Wang, \& Li 2011). The relatively high luminosity range $\left(L_{X} \gtrsim 10^{31}\right)$ should be dominated by CVs. The specific population of such sources in a GC depends on its dynamical state and can be greater than that in the Galactic bulge or in the solar neighborhood. Interestingly, the source population, particularly in the lower luminosity
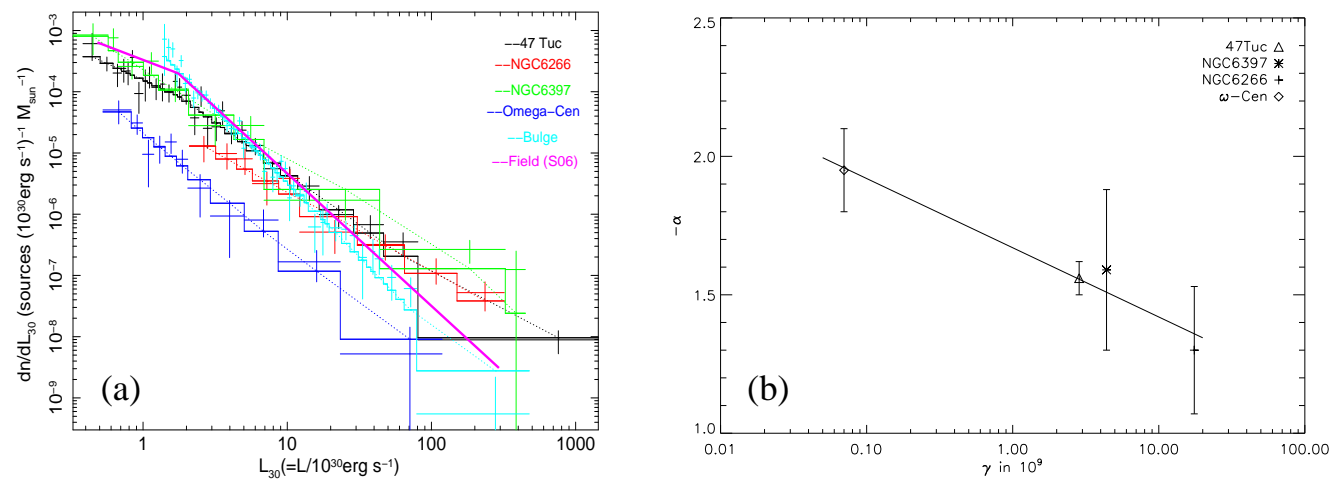

Figure 1. (a) Specific XLFs of four GCs and a Galactic bulge field, as well as the best-fit broken power law model for sources in the solar neighborhood (Field; Xu, Wang, \& Li 2011). The incompleteness and bias of the source detection have been approximately corrected, while the contributions from interlopers have been subtracted statistically from the data. (b) Correlation between the power law slopes of the LFs and the mass-averaged encounter rate $(\gamma)$ of the GCs, demonstrating the importance of the dynamical effect on formation and evolution of binaries. 
range, is generally smaller in the GCs (especially in those dynamically less evolved ones) than in the bulge and field. To explain this result, one probably needs to invoke both the dynamical formation of binaries and the assumption that the initial binary fraction of stars is very small in GCs, consistent with existing optical observations (e.g., Davis et al. 2008). The dynamical effect has also been shown to be important in explaining the increasing specific LMXB population in the central bulge of M31 (Zhang et al. 2011). In regions outside of GCs and Galactic nuclear regions, however, we can now reasonably determine and subtract the faint stellar X-ray contribution (Revnivtsev et al. 2008; Zhang et al. 2011) to facilitate the measurements of truly diffuse X-ray emission from galaxies.

\section{Diffuse Soft X-ray Emission}

Diffuse soft X-ray emission has commonly been used to trace various types of galactic feedback in nearby starburst and normal galaxies, as well as the cooling of hot gaseous halos or coronae resulting from the galaxy formation (accretion) processes (e.g, Crain et al. 2010). Assuming an origin of this emission in optically-thin thermal (collisionallyexcited) hot plasma, one may estimate its mass, energy, and chemical contents and even their outflow or accretion rates from a galaxy.

Emission from Galactic Spheroids. While galactic coronae associated with giant elliptical galaxies have been studied extensively (e.g, Sun et al. 2007; Mulchaey \& Jeltema 2010; Pellegrini 2011), the very detection of truly diffuse X-ray emission in and around low- $L_{x}$ stellar spheroids (galactic bulges or low- to intermediate-mass ellipticals) becomes possible only recently, thanks largely to the calibration of the stellar $L_{x}$ to mass ratio, as discussed above. A good example of such a detection is the bulge of M31 (Fig 2 $\mathrm{b}$; Li et al. 2007; Bogdán \& Gilfanov 2011). The large-scale X-ray emission shows a bipolar morphology along the minor axis of the galaxy, indicating an outflow of hot plasma, which is most likely driven by Ia SNe because there is essentially no AGN and no recent SF in the bulge. But the X-ray luminosity accounts for only $\sim 2 \%$ of the Ia SN mechanical energy input in the bulge. There is no bulge-wide distributed cool gas to potentially consume or convert the energy into other forms. Therefore, the outflow is actually required to explain the lack of the accumulation of the stellar feedback, not only the energy, but the mass (mainly from redgiant winds and planetary nebulae) and the iron (from Ia SNe, about $0.7 M_{\odot}$ each) as well. Theoretically, it has been shown that such outflows play an important role in maintaining large-scale hot gaseous halos around galactic spheroids (Tang et al. 2009).

The dynamical state of a corona around a galactic spheroid depends on its gravitational mass and environment (e.g., Wang et al. 2004; Owen et al. 2006; Sun et al. 2007, 2010; Mulchaey \& Jeltema 2010; Weźgowiec et al. 2011; Tang et al. 2009; Lu \& Wang 2011). The corona of an intermediate-mass spheroid fast moving in a rich cluster, for example, most likely represents an Ia SN-driven outflow semi-confined by the ram pressure of the intracluster medium (ICM; Lu \& Wang 2011). The ram pressure affects the size and lopsidedness of the coronae. Because of the semi-confinement, the outflow should also be subsonic. The hot gas density increases with the ICM thermal pressure, which may lead to the compression of cool gas clouds, if present, and hence the formation of stars (Young \& Scoville 1991; Bekki \& Couch 2003). Such density increase also enhances radiative cooling of the hot gas, which may fuel central supermassive black holes, explaining why the frequency of active galactic nuclei observed in clusters tends to be higher than that in the field (e.g., Hart, Stocke \& Hallman 2009) and is apparently responsible for a substantially higher surface brightness of the X-ray emission detected from corona in the cluster environment. Furthermore, the total X-ray luminosity of a corona depends on the 
relative importance of the surrounding thermal and ram pressures. These environment dependencies should at least partly explain the large dispersion in the observed diffuse X-ray luminosities of spheroids with similar stellar properties. Furthermore, an outflow powered by the distributed feedback can naturally produce a positive radial gradient in the hot gas entropy, mimicking a cooling flow.
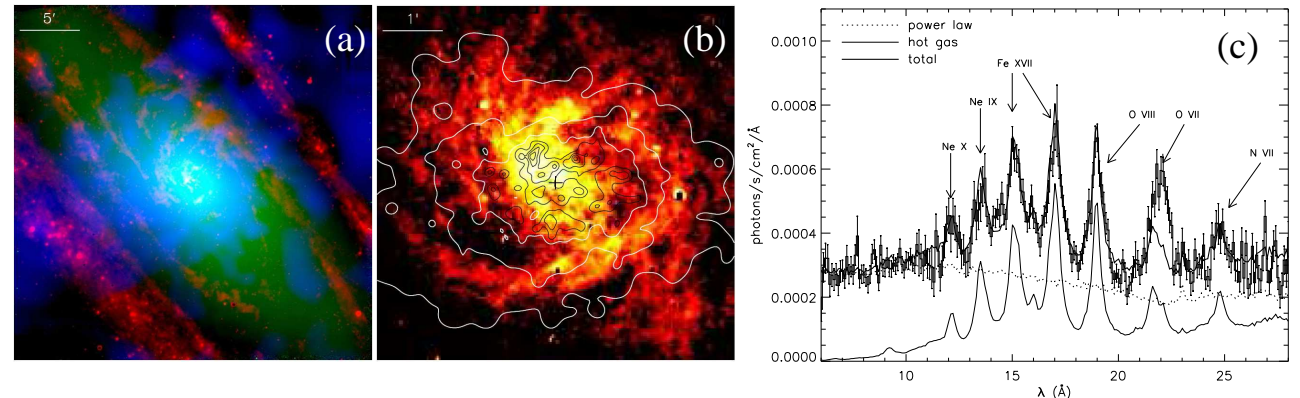

Figure 2. (a) Tri-color image of the spheroid region of M31: Spitzer/MIPS $24 \mu \mathrm{m}$ emission (red), 2MASS K-band emission (green), and Chandra 0.5-2 keV emission of truly diffuse hot gas (blue; Li \& Wang 2007). (b) Intensity contours of the diffuse emission overlaid on an $\mathrm{H}_{\alpha}$ image in the M31 central region. A detailed analysis of the data shows evidence for strong interaction between the hot and cool gas phases, which may provide a mechanism for starving the nucleus as marked by the plus sign (Li et al. 2009). (c) An XMM-Newton Reflection Grating Spectrometer (RGS) spectrum of the M31 central region (Liu et al. 2010). The curves show the fit with a single-temperature thermal plasma, plus a power-law that characterizes the discrete source contribution. Note the intensity excess of the $\mathrm{O}$ VII $\mathrm{K} \alpha$ triplet above the model, giving a strong indication for emission from the CX between hot ions and neutral atoms.

However, the soft X-ray emission in the inner regions of spheroids is still far from being well understood. For example, the low temperature and metal abundance of hot plasma, as inferred from the existing spectral studies, are still difficult to be reconciled with models and simulations (e.g., Tang \& Wang 2010). Even the very assumption that the X-ray line emission originates predominantly in optically-thin thermal plasma is problematic (Liu et al. 2010). This becomes apparent when the so-called $\mathrm{G}[=(f+i) / r$, forbidden+intercombination to resonance line] ratio of the $\mathrm{K} \alpha$ triplet of such a diagnostic He-like ion as $\mathrm{O}$ VII is analyzed. For an optically-thin thermal plasma with a temperature of a few $10^{6} \mathrm{~K}$ and in collisional ionization equilibrium, G should be considerably less than one (e.g., Porquet et al. 2010). Fig. 2r presents an RGS spectrum of the M31 central region (Fig 2b), showing a clear intensity excess above a simple thermal plasma model fit at $\sim 22 \AA$. This excess is due to the presence of the strong forbidden line (at $22.1 \AA$ ), relative to the resonant one $(21.6 \AA)$ of the $\mathrm{O}$ VII $\mathrm{K} \alpha$ triplet. Furthermore, the excess is shown to extend throughout much of the cross-dispersion range of the RGS observations and seems to be correlated well with the distribution of the cool gas (Fig. $2 \mathrm{~b}$ ). One natural explanation is that the excess or the strong forbidden line represents the contribution from the CX between the two gas phases.

Emission from Active SF Galaxies. Similar conclusions are also reached in studies of diffuse soft X-ray emission from active SF galaxies. Such emission has been mapped out for many face-on galaxies (e.g., Tyler et al. 2003; Doane et al. 2004; Owen \& Warwick 2009; Kuntz \& Snowden 2010). The emission is closely correlated with recent SF. The study of M101, for example, shows that the bulk ( $\gtrsim 80 \%)$ of the emission is associated with SF regions with ages $\lesssim 20 \mathrm{Myr}$, but is not due to individual SNRs, as identified in optical and radio (e.g., Kuntz \& Snowden 2010). The emission traces diffuse hot plasma, which can naturally be heated by fast stellar winds and SNe of massive stars. This is 
most vividly demonstrated by the emission from within cool gas cavities around massive stellar clusters, as in the 30 Doradus nebula (Wang et al. 1999). The X-ray CCD spectra of the emission from galaxies can typically be approximately fitted with an opticallythin thermal plasma model with two characteristic temperatures of $\sim 0.2 \pm 0.1$ and $0.7 \pm 0.1 \mathrm{keV}$; the low-temperature component typically accounts for the bulk of the observed flux. However, the total luminosity of the emission accounts for a very small fraction $(\lesssim 1 \%)$ of the expected mechanical energy input rate from fast stellar winds and $\mathrm{SNe}$ of massive stars. The estimated thermal energy of the plasma is also substantially smaller than the expected total energy input. Although a fraction of the energy may be carried away by cosmic rays or converted to radiation in other wavelength bands, outflows from recent SF regions (i.e., blown-out superbubbles or galactic chimneys) are expected, venting chemically-enriched hot plasma into the large-scale halos of galaxies. Indeed, extraplanar soft X-ray emission is observed around active SF galaxies (Wang et al. 2001, 2003; Tüllmann 2006; Wang 2010 and references therein; Li \& Wang 2011; Anderson \& Bregman 2011). Such emission also often appears to be correlated with extraplanar $\mathrm{H} \alpha$-emitting and/or dusty features. This correlation has led to the conclusion that the bulk of the emission arises from the interaction between the outflowing hot plasma and entrained or pre-existing cool gas clouds, rather than from the superwinds themselves (e.g., Strickland \& Heckman 2009).

To determine the nature of the interaction, we have recently examined the spectroscopic properties of the soft X-ray emission from nine nearby active SF galaxies, which show no significant AGN activities (Liu et al. 2011a,b), using high spectral resolution RGS data with good signal-to-noise ratios. The RGS spectra of these galaxies typically show large G ratios of the OVII K $\alpha$ triplet, indicating that a substantial fraction of the emission cannot simply arise from collisionally-excited hot plasma, as commonly assumed. The bulk of the OVII $\mathrm{K} \alpha$ line emission is consistent with an origin in the CX between highly ionized ions (i.e., OVIII) and neutral atoms (e.g., HI and HeI); other possible mechanisms for producing a high $\mathrm{G}$ ratio, such as a collisional non-equilibrium-ionization recombining/ionizing plasma, are not favored, although they cannot be completely ruled out. In the central region of M82, for example, the putative CX contribution to the key He-like K $\alpha$ triplets is on average $\gtrsim 50 \%$, decreasing with the increasing ionization states (i.e., the $\mathrm{G}$ ratio is smaller for NeIX and Mg XI than for OVII; Liu et al. 2011a). To quantify the true contribution of the CX, one needs to have a spectral model of it, which is not yet available. Nevertheless, the joint analysis of multiple emission lines indicates that the thermal plasma is substantially hotter, probably consistent with the high-temperature component indicated in the 2-temperature characterization mentioned above. The decomposition of the CX and thermal contributions will also be essential to a correct estimation of the metal abundances of the plasma (Liu et al. 2011a).

The low X-ray emission efficiency of the galactic feedback $(\sim 1 \%)$ has strong implications for understanding the formation and evolution of galaxies. The efficiency shows no significant correlation with the stellar mass or gas content of a galaxy (Li \& Wang 2011). There is also little evidence that the feedback energy is consumed at other wavelengths. For example, Grimes et al. (2009) show that O VI emission is not generally detected in their sample of starburst galaxies, suggesting that radiative cooling or turbulent mixing is not significant in draining energy from galactic winds. One may thus conclude that the bulk of the energy is gone with the winds and is released to the halos of galaxies, a topic that certainly deserves further attention. 


\section{Summary and Conclusions}

I have reviewed some basic properties of various types of X-ray sources, particularly in relation to the spectral energy distribution (SED) of galaxies. The key points are as follows:

- AGNs are typically the brightest X-ray sources in galaxies and can be detected relatively easily even at high redshifts with little confusion. Such detection is important in modeling the SED of galaxies. Even low- $L_{x}$ AGNs, as typically seen in nearby galaxies, can be important in regulating galactic nuclear environments.

- In nearby galaxies, the bulk of HMXBs and LMXBs can readily be detected. Their overall galactic populations are well correlated with the SFR and stellar mass and can also be independently estimated (e.g., with infrared observations). The comparisons of these independent estimates can then be used to constrain the presence of AGNs in distant galaxies, even if they are not well resolved.

- The X-ray source population of a stellar system is particularly sensitive to its binary fraction. Thus X-ray observations provide a powerful tool to probe the initial binary fraction and dynamical evolution of stellar clusters and even galactic spheroids.

- Diffuse X-ray emission traces the galactic feedback, primarily due to massive SF (via fast stellar winds and core-collapsed $\mathrm{SNe}$ ) in galactic disks, to old stars (mass-loss and Ia SNe), and to possible AGNs. However, the luminosity of the X-ray emission from a galaxy typically accounts for $\lesssim 1 \%$ of the feedback energy. The bulk of the energy is released in outflows of hot plasma and cosmic-rays/magnetic fields from stellar spheroids, as well as from SF regions.

- A substantial fraction of the diffuse X-ray emission may arise from the CX at the interface between hot plasma and cool gas. The CX, in principle, can be used as a new tool to probe the thermal, chemical, and kinematic properties of hot plasma tracing various high-energy feedback processes in the galaxies.

While much can still be done with the existing X-ray observatories, we will also have new tools in the near future. NuSTAR (scheduled to be launched in late 2012) will offer the first imaging capability in the 8-80 keV range, while Astro- $H$ (2013) will provide the calorimeter-based imaging spectroscopic capability in the $0.5-10 \mathrm{keV}$ range. Furthermore, eROSITA (2013) will conduct the first all-sky X-ray CCD imaging survey over the 0.3$10 \mathrm{keV}$ range, while GEMS (2014) will enable polarization measurements in the 2-10 $\mathrm{keV}$ range. With these new capabilities, we can expect a substantial advance in our understanding of X-ray sources in galaxies.

I am grateful to the organizers of the IAU Symposium for inviting me to give a review talk on this topic, which the present article is based on, and to my students and collaborators for their contributions to some of the work described above. My research is largely supported by NASA via various grants.

\section{References}

Anderson, M.E., \& Bregman, J.N. 2011, ApJ, 737, 22

Bekki, K., \& Couch, W.J., 2003, ApJ, 596, 13

Bell, E.F. \& De Jong, R.S. 2001, ApJ, 550, 212

Bogdán, A. \& Gilfanov M. 2011, MNRAS, in press

Crain, R.A., et al. 2010, MNRAS, 407, 1403

Davis, D. S., Richer, H. B., Anderson, J., et al. 2008, AJ, 135, 2155

Dijkstra, M. et al. 2011, MNRAS, submitted (arXiv1108.4420)

Doane, N.E., et al. 2004, AJ, 128, 2712

Gilfanov, M. 2004, MNRAS, 349, 146 
Grimes, J.P., et al. 2009, ApJS, 181, 272

Grimm, H.-J., Gilfanov M., \& Sunyaev R., 2003, MNRAS, 339, 793

Guainazzi, M. \& Bianchi, B. 2007, MNRAS, 374, 1290

Hart, Q.N., Stocke, J.T., \& Hallman, E.J., 2009, ApJ, 705, 854

Irwin, J.A., Athey, A.E., \& Bregman, J.N. 2003, ApJ, 587, 356

Kim, D.-W. \& Fabbiano, G. 2004, ApJ, 611, 846

Kim, D.-W. \& Fabbiano, G. 2010, ApJ, 721, 1523

Kuntz, K.D., \& Snowden, S.L. 2010, ApJS, 188, 46

LaMassa, S.M. 2009, ApJ, 705, 568

Li, Z., \& Wang, Q.D. 2007, ApJL, 668, 39

Li, Z., Wang, Q.D. \& Wakker, B.P. 2009, MNRAS, 397, 148

Li, Z., et al. 2011, ApJL, 728, 10

Li, J., \& Wang, Q.D. 2011, MNRAS, submitted

Liu, J., Wang, Q.D., Li Z., \& Peterson J.R. 2010, MNRAS, 404, 1879

Liu, J., Mao, S., \& Wang, Q.D. 2011a, MNRAS, 415, 64

Liu, J., Wang, Q.D., \& Mao, S. 2011b, MNRAS, in press (arXiv:1111.5915)

Lu, Z., \& Wang Q.D. 2011, MNRAS, 413, 347

Mineo, S., Gilfanov, M., \& Sunyaev, R. 2011, MNRAS, in press (arXiv:1105.4610v2)

Mulchaey, J.S., \& Jeltema, T.E. 2010, ApJL, 715, 1

Omma, H., Binney, J., Bryan, G., \& Slyz, A. 2004, MNRAS, 348, 1105

Oppenheimer, B.D. 2010, MNRAS, 406, 2325

Owen, F., et al. 2006, AJ, 131, 1974

Owen, R.A., \& Warwick, R.S. 2009, MNRAS, 394, 174

Pellegrini, S., 2011, ApJ, 738, 57

Pellegrini, S., Ciotti, L., \& Ostriker, J.P. 2011, ApJ, submitted (arXiv1107.3675)

Persic, M., \& Rephaeli, Y. 2007, A\&A, 463, 481

Porquet, D., Dubau, J. \& Grosso, N. 2010, SSRv, 157, 103

Ranalli, P., et al. 2008, MNRAS, 386, 1464

Revnivtsev, M. et al. 2008, A\&A, 490, 37

Revnivtsev, M., et al. 2011, A\&A, 526, 94

Sazonov, S., et al. 2006, A\&A...450..117

Strickland, D., \& Heckman, T. 2009, ApJ, 697, 2030

Sun, M., et al. 2007, ApJ, 657, 197

Sun, M., et al. 2010, 2010, ApJ, 708, 946

Swartz, D.A., et al. 2011, ApJ, 741, 49

Tang, S., et al. 2009, MNRAS, 392, 77

Tang, S., \& Wang, Q.D. 2010, MNRAS, 408, 1011

Townsley, L.K., et al. 2011, ApJS, 194, 16

Tüllmann, R., et al. 2006, A\&A, 457, 779

Tyler, K., et al. 2003, ApJ, 610, 213

Voss, R., et al. 2009, ApJ, 701, 474

Wang, J., et al. 2010, ApJ, 719, 208

Wang, Q.D. 1999, ApJL, 510, 139

Wang, Q.D., et al. 2001, ApJ, 555, 99

Wang, Q.D., Chaves, T. \& Irwin, J.A. 2003, ApJ, 598, 969

Wang, Q.D., Owen, F. \& Ledlow, M. 2004, ApJ, 611, 821

Wang, Q.D. 2010, PNAS, 107, 7168

Weźgowiec, M., et al. 2011, A\&A, 531, 44

White, N.E., Swank, J.H., \& Holt, S.S. 1983, ApJ, 270, 711

Xu, X., Wang, Q.D., \& Li, X.-D. 2011, in preparation

Young, J.S., \& Scoville, N.Z., 1991, ARA\&A, 29, 581

Yuan, F., Xie, F., \& Ostriker, J.P. 2009, ApJ, 691, 98

Yuan, F. 2007, ASPC, 373, 95

Zhang, Z., et al. 2011, A\&A, 533, 33 